OPEN ACCESS

Edited by:

Roberto Pola,

Università Cattolica del Sacro

Cuore, Italy

Reviewed by:

Håkan Wallen,

Karolinska Institutet (KI), Sweden

Mettine H.A. Bos,

Leiden University Medical

Center, Netherlands

${ }^{*}$ Correspondence:

Chien-Ning Hsu

chien_ning_hsu@hotmail.com orcid.org/0000-0001-7470-528X

Specialty section:

This article was submitted to

Thrombosis,

a section of the journal

Frontiers in Cardiovascular Medicine

Received: 03 August 2021

Accepted: 21 September 2021

Published: 18 October 2021

Citation:

Fu C-M, Li L-C, Lee Y-T, Wang S-W and Hsu C-N (2021) Apixaban vs. Warfarin in Atrial Fibrillation Patients With Chronic Kidney Disease.

Front. Cardiovasc. Med. 8:752468. doi: 10.3389/fcvm.2021.752468

\section{Apixaban vs. Warfarin in Atrial Fibrillation Patients With Chronic Kidney Disease}

\author{
Chung-Ming Fu ${ }^{1}$, Lung-Chih $\mathrm{Li}^{1,2}$, Yueh-Ting Lee ${ }^{1}$, Shih-Wei Wang ${ }^{3}$ and Chien-Ning Hsu ${ }^{3,4 *}$ \\ ${ }^{1}$ Division of Nephrology, Department of Internal Medicine, Kaohsiung Chang Gung Memorial Hospital, Chang Gung University \\ College of Medicine, Kaohsiung, Taiwan, ${ }^{2}$ Institute for Translational Research in Biomedicine, Kaohsiung Chang Gung \\ Memorial Hospital, Chang Gung University College of Medicine, Kaohsiung, Taiwan, ${ }^{3}$ Department of Pharmacy, Kaohsiung \\ Chang Gung Memorial Hospital, Kaohsiung, Taiwan, ${ }^{4}$ School of Pharmacy, Kaohsiung Medical University, Kaohsiung, Taiwan
}

Background and Objectives: Real-world evidence of apixaban treatment in patients with chronic kidney disease remains scarce. This study aimed to compare the relative risk of stroke or systemic embolism (SE) and major bleeding between apixaban and warfarin in atrial fibrillation (AF) patients with different degrees of kidney function.

Design, Setting, Participants, and Measurements: We evaluated newly diagnosed AF patients between 2004 and 2018, who were receiving apixaban or warfarin. Electronic medical record data were collected from a large healthcare delivery network in Taiwan. The outcomes of hospitalization for stroke/SE and major bleeding were compared with propensity-score matched apixaban and warfarin cohorts. Stratified analyses according to initial apixaban dose (standard dose of $10 \mathrm{mg} /$ day vs. lower dose of 2.5-5.0 mg/day) and baseline estimated glomerular filtration rate were performed.

Results: Each cohort involved 1,625 matched patients. Apixaban was significantly associated with a lower risk of stroke/SE (adjusted hazard ratio [aHR]: 0.74; 95\% confidence interval [Cl]:0.57-0.97; $p=0.03$ ). The risk of major bleeding was not increased whether in standard doses (aHR: 0.66; 95\% Cl: 0.45-0.96; $p=0.03$ ) or reduced doses (aHR, 0.84; 95\% Cl, 0.63-1.12; $p=0.23$ ) of apixaban. Regarding kidney function, apixaban reduced the risk of stroke/SE by $37 \%$ in those with an eGFR of $<30$ $\mathrm{ml} / \mathrm{min} / 1.73 \mathrm{~m}^{2}$ (aHR: 0.63; 95\% Cl: 0.40-0.98; $p=0.04$ ).

Conclusions: Compared to warfarin, apixaban is associated with a reduced risk of stroke/SE and is consistent with a subset of AF patients with eGFR $<30 \mathrm{ml} / \mathrm{min} / 1.73$ $\mathrm{m}^{2}$. Both standard and reduced doses of apixaban showed lower risk of major bleeding than those of warfarin.

Keywords: apixaban, warfarin, chronic kidney disease, atrial fibrillation (AF), ischemic stroke, bleeding, thromboembolism 


\section{INTRODUCTION}

Atrial fibrillation (AF) as the most common cardiac arrhythmia (1) and contributes significantly to cerebral ischemic stroke and other severe thromboembolic events. To prevent these severe complications, current guidelines stipulate that high-risk AF patients $\left(\mathrm{CHA}_{2} \mathrm{DS}_{2}\right.$ - VASc scores $\geq 2$ ) should be prescribed direct oral anticoagulants (DOACs) rather than vitamin $\mathrm{K}$ antagonists (2-5). Patients with chronic kidney disease (CKD) have a 2to 3 -fold higher prevalence of AF than the general population (6-8). In addition, CKD itself contributes to a pro-thrombotic state, which increases the risks of ischemic stroke or systemic embolism (9-11). The risk of thromboembolic events is even worse in $\mathrm{CKD}$ patients receiving renal replacement therapy $(11,12)$. Furthermore, patients with an estimated glomerular filtration rate (eGFR) of $<30 \mathrm{~mL} / \mathrm{min} / 1.73 \mathrm{~m}^{2}$ have a higher risk of bleeding compared to those with an eGFR of between 30 and $60 \mathrm{~mL} / \mathrm{min} / 1.73 \mathrm{~m}^{2}$ and those with an eGFR $\geq 60 \mathrm{~mL} / \mathrm{min} / 1.73$ $\mathrm{m}^{2}$ while receiving oral anticoagulant (OAC) therapy (12-14). Importantly, most pivotal studies of DOACs excluded patients with advanced CKD and end-stage kidney disease (ESKD). Thus, real-world evidence is needed to optimize the prevention of thromboembolism, and still minimize the risk of bleeding in patients with abnormal kidney function.

A patient's kidney function is one of the factors that influences OAC selection $(15,16)$, and warfarin is often prescribed in patients with CKD. Apixaban is currently the only approved $\mathrm{DOAC}$ for $\mathrm{AF}$ patients with serum creatinine clearance $(\mathrm{CrCl})$ of $<15 \mathrm{~mL} / \mathrm{min}$; however, approval was based on a pharmacokinetic study of only eight patients with CKD on dialysis (17). Furthermore, treatment outcomes of apixaban in ESKD patients have been reported $(18,19)$. A study using 2010-2015 Renal Data System in the United State (USRDS) data found that although apixaban has no benefit on stroke/systemic embolism (SE) prevention, it is associated with a significantly lower risk of major bleeding compared to warfarin (18). Another study using USRDS data (2012-2015) compared apixaban with no anticoagulation in patients with chronic dialysis and AF, and found that apixaban treatment was not associated with risk reductions in both ischemic stroke and fatal or intracranial bleeding (19). Given that these studies mainly focused on the necessity of anticoagulation in the chronic dialysis population, the usefulness of apixaban treatment in CKD patients without dialysis treatment is still unclear. Thus, this study aimed to compare the relative risk of stroke or SE and major bleeding between apixaban and warfarin in AF patients with different degrees of kidney disease.

\section{MATERIALS AND METHODS \\ Study Design and Data Source}

This was a retrospective cohort study of adult patients with non-valvular AF or atrial flutter. Data were obtained from the Chang Gung Research Database (CGRD), a de-identified, electronic health records database of patient information from the healthcare delivery system in Taiwan. The CGRD contains
International Classification of Diseases, Ninth/Tenth Revision, Clinical Modification (ICD-9/10-CM) codes, Healthcare Common Procedure Coding System codes, Anatomical Therapeutic Chemical Classification System codes, and laboratory test results in emergency departments and inand-outpatient settings (Supplementary Table 1). The data sets and have been described previously $(20,21)$.

This study was approved by the Institutional Review Board of Chang Gung Medical Foundation at Taipei, Taiwan (approval number 201900901B0) and was conducted according to the tenets of the Declaration of Helsinki. The need for informed consent was waived owing to the retrospective nature of the study.

\section{Patients}

We evaluated AF patients who were newly diagnosed between January 1, 2004, and December 21, 2018, in whom apixaban or warfarin therapy had been initiated. The inclusion criteria were having a diagnosis of AF before the index date (the date of apixaban or warfarin initiation) and at least one or more admissions within at least 12 months before the index date (Figure 1). Patients were excluded if they had any of the following: OAC treatment (warfarin, apixaban, dabigatran, rivaroxaban, edoxaban) within 3 months before the index date, missing serum creatinine ( $\mathrm{SCr}$ ) results, moderate or severe mitral stenosis, valve replacement, peritoneal dialysis, or kidney transplantation. The patient selection criteria are detailed in Figure 1, Supplementary Table 1. The patients were identified using ICD-9/10-CM codes on at least two outpatient visits with an interval of more than 28 days or on one postdischarge follow-up within the study period. The first apixaban or warfarin prescription date in the outpatient setting was designated as the index date for patients without any other OAC treatment.

\section{Outcome Measures}

Effectiveness was evaluated according to the incidence of stroke or SE as outcome measure, while safety was evaluated according to the incidence of major bleeding, including any intracranial hemorrhage but not traumatic hemorrhage, intraabdominal, gastrointestinal bleeding, hematuria, or bleeding at other sites (Supplementary Table 1) (22). The outcomes of interest were defined according to discharge diagnosis in the as-treated cohort. All patients were followed up from the index date to the first event of interest, discontinuation date of apixaban or warfarin, medication switch date, in-hospital death, loss to follow-up ( $\geq 365$ days without any medical encounters before the end date of the database), or the data cut-off date (December 31, 2018), whichever came first.

\section{Statistical Analysis}

The patients were matched using propensity scores (PS) to minimize selection bias $(23,24)$. Each patient's PS was calculated based on the following characteristics: demographic data, such as age and sex; individual disease condition in the Charlson Comorbidity Index (25); eGFR; hypertension; major bleeding; medications; $\mathrm{CHA}_{2} \mathrm{DS}_{2}$-VASc score and HAS-BLED score. The 


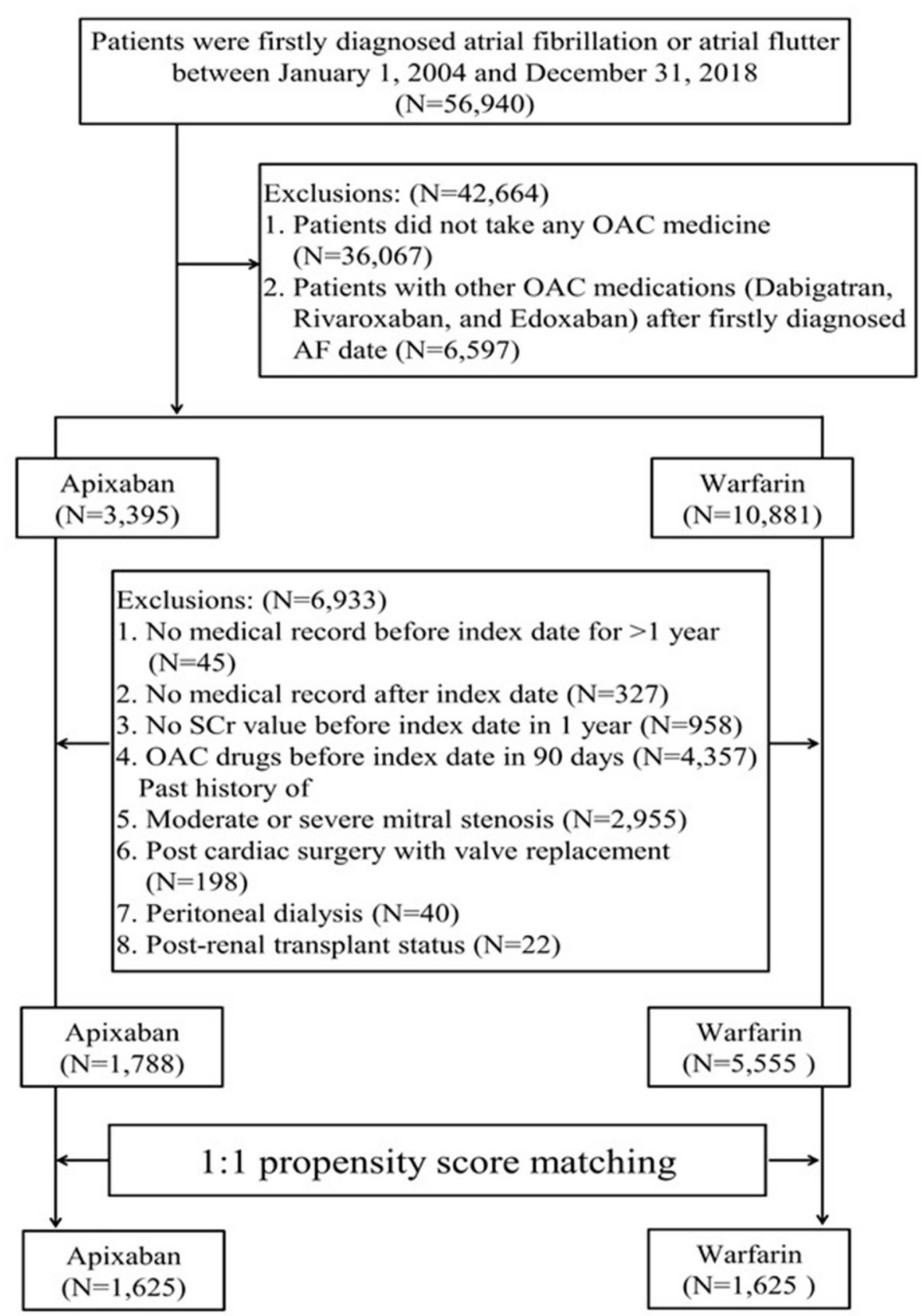

FIGURE 1 | Patient inclusion flowchart.

covariates for the PS matching model are presented in Table 1, Supplementary Table 1. Patients who were prescribed apixaban or warfarin were matched in a 1:1 ratio using a greedy algorithm (26). The distributions of PS was assessed, and the covariate balance between treatment groups were determined using the standardized mean differences (SMD) with a threshold of $<10 \%$ (27). The relative risk of stroke/SE and major bleeding between apixaban and warfarin was evaluated using Cox proportional hazards models separately, and adjusted for covariates listed in Table 1.

Subgroup analysis according to the apixaban dose (standard dose $[10 \mathrm{mg} /$ day $]$ vs. reduced dose [2.5-5.0 $\mathrm{mg} /$ day] $)$ was conducted using the Cox proportional hazards model to evaluate the dose relationship with the heterogeneity of treatment effects. Subgroup analysis according to baseline eGFR ( $\geq 60 \mathrm{ml} / \mathrm{min} / 1.73$ $\mathrm{m}^{2}$ (mild CKD), 59.9-30.0 $\mathrm{ml} / \mathrm{min} / 1.73 \mathrm{~m}^{2}$ (moderate CKD), 
TABLE 1 | Baseline patient characteristics by treatment group before and after propensity score matching.

\begin{tabular}{|c|c|c|c|c|c|c|}
\hline & \multicolumn{3}{|c|}{ Before propensity score matching } & \multicolumn{3}{|c|}{ After propensity score matching } \\
\hline$<40$ & $122(2.20)$ & $12(0.67)$ & 0.129 & $11(0.68)$ & $12(0.74)$ & 0.007 \\
\hline $40-64$ & $1,882(33.88)$ & $250(13.98)$ & 0.480 & $257(15.82)$ & $245(15.08)$ & 0.020 \\
\hline $65-74$ & $1,578(28.41)$ & $537(30.03)$ & 0.036 & $503(30.95)$ & $504(31.02)$ & 0.001 \\
\hline \multicolumn{7}{|l|}{ Sex, $n(\%)$} \\
\hline Male & $3,335(60.04)$ & $1,023(57.21)$ & 0.057 & $926(56.98)$ & $938(57.72)$ & 0.015 \\
\hline Female & 2,220 (39.96) & 765 (42.79) & 0.057 & $699(43.02)$ & $687(42.28)$ & 0.015 \\
\hline \multicolumn{7}{|c|}{ Baseline eGFR, $\mathrm{ml} / \mathrm{min} / 1.73 \mathrm{~m}^{2}, n(\%)$} \\
\hline$\geqq 90$ & $1,001(18.02)$ & $286(16.00)$ & 0.054 & $286(17.60)$ & $277(17.05)$ & 0.015 \\
\hline$<15$ & $311(5.60)$ & $16(0.89)$ & 0.268 & $14(0.86)$ & $16(0.98)$ & 0.013 \\
\hline \multicolumn{7}{|c|}{ Charlson comorbid conditions, $\boldsymbol{n}(\%)$} \\
\hline Acute myocardial infarction & $354(6.37)$ & $103(5.76)$ & 0.026 & $94(5.78)$ & $95(5.85)$ & 0.003 \\
\hline Congestive heart failure & 1,857 (33.43) & $568(31.77)$ & 0.036 & 537 (33.05) & 519 (31.94) & 0.024 \\
\hline Peripheral vascular diseases & $245(4.41)$ & $44(2.46)$ & 0.107 & $29(1.78)$ & $44(2.71)$ & 0.062 \\
\hline Cerebral vascular accident & 2,013 (36.24) & 589 (32.94) & 0.069 & $505(31.08)$ & 525 (32.31) & 0.026 \\
\hline Dementia & $135(2.43)$ & $51(2.85)$ & 0.026 & 49 (3.02) & 48 (2.95) & 0.004 \\
\hline Pulmonary disease & $1,031(18.56)$ & $291(16.28)$ & 0.060 & 261 (16.06) & $277(17.05)$ & 0.027 \\
\hline Connective tissue disorder & $26(0.47)$ & $17(0.95)$ & 0.058 & $14(0.86)$ & $14(0.86)$ & 0.000 \\
\hline Peptic ulcer & 766 (13.79) & $291(16.28)$ & 0.070 & $270(16.62)$ & $266(16.37)$ & 0.007 \\
\hline Metastatic cancer & $59(1.06)$ & $30(1.68)$ & 0.053 & $25(1.54)$ & $28(1.72)$ & 0.015 \\
\hline Hypertension & $3,612(65.02)$ & $1,314(73.49)$ & 0.184 & $1,186(72.98)$ & $1,177(72.43)$ & 0.012 \\
\hline Prior major bleeding & $1,147(20.65)$ & $582(32.55)$ & 0.272 & $479(29.48)$ & $484(29.78)$ & 0.007 \\
\hline \multicolumn{7}{|l|}{ Prior medication uses } \\
\hline Lipid-lowering agent & 905 (16.29) & $485(27.13)$ & 0.265 & $409(25.17)$ & $408(25.11)$ & 0.001 \\
\hline Glucose-lowering agent & 1,088 (19.59) & 403 (22.54) & 0.073 & 342 (21.05) & 340 (20.92) & 0.003 \\
\hline Anti-hypertension & $3,857(69.43)$ & 1,394 (77.96) & 0.195 & $1,262(77.66)$ & $1,246(76.68)$ & 0.024 \\
\hline Anti-platelet agent & $2,433(43.80)$ & $753(42.11)$ & 0.034 & $708(43.57)$ & $690(42.46)$ & 0.022 \\
\hline Aspirin & 2,029 (36.53) & $560(31.32)$ & $<0.0001$ & $583(35.88)$ & $512(31.51)$ & - \\
\hline Clopidogrel & $552(9.94)$ & $261(14.6)$ & $<0.0001$ & $171(10.52)$ & $238(14.65)$ & - \\
\hline Ticagrelor & $12(0.22)$ & $14(0.78)$ & $<0.0001$ & $3(0.18)$ & $11(0.68)$ & - \\
\hline Others & $264(4.75)$ & $67(3.75)$ & 0.112 & $72(4.43)$ & $60(3.69)$ & - \\
\hline Amiodarone & $841(15.14)$ & 355 (19.85) & 0.124 & 302 (18.58) & $301(18.52)$ & 0.002 \\
\hline Digoxin & $839(15.10)$ & 150 (8.39) & 0.210 & $149(9.17)$ & $146(8.98)$ & 0.006 \\
\hline NSAIDs & $568(10.23)$ & 200 (11.19) & 0.031 & $189(11.63)$ & $185(11.38)$ & 0.008 \\
\hline Gastric antacids & $922(16.60)$ & $506(28.30)$ & 0.283 & $421(25.91)$ & $423(26.03)$ & 0.003 \\
\hline \multicolumn{7}{|l|}{ Mean value (SD) } \\
\hline $\mathrm{CHA}_{2} \mathrm{DS}_{2}$-VASc score & $3.40(1.84)$ & $3.92(1.70)$ & 0.293 & $3.81(1.69)$ & $3.83(1.68)$ & 0.011 \\
\hline HAS-BLED score & $2.55(1.43)$ & $3.02(1.36)$ & 0.333 & $2.92(1.36)$ & $2.92(1.34)$ & 0.003 \\
\hline
\end{tabular}

SMD, standardized mean difference; SD, standard deviation; CKD, chronic kidney disease; eGFR, estimated glomerular filtration rate; NSAID: non-steroidal anti-inflammatory drug. 
and $<30 \mathrm{ml} / \mathrm{min} / 1.73 \mathrm{~m}^{2}$ (advanced CKD) was also performed to evaluate the influence of kidney function on the effectiveness of apixaban and warfarin. Baseline eGFR was calculated based on the mean serum creatinine ( $\mathrm{SCr}$ ) level within 3 months prior to the index date and using the Modification of Diet in Renal Disease (MDRD) equation (28): $175 \times \mathrm{SCr}(\mathrm{mg} / \mathrm{dL})^{-1.154}$ $\times$ age (years) ${ }^{-0.203} \times 0.742$ (if female). Furthermore, hospital admissions for pneumonia or hip fracture were regarded as negative control outcomes (19) to ensure the robustness of the study results. We hypothesized that pneumonia and hip fracture had the same exposure risk in the apixaban and warfarin groups. All statistical analyses were performed using SAS 4.0 (Cary, NC, USA). A two-sided $P$-value of $<0.05$ was considered statistically significant.

\section{RESULTS}

\section{Patient Characteristics}

A total of 56,940 patients with AF or atrial flutter diagnosis were identified. Out of them, 7,343 patients who were administered warfarin $(n=5555)$ or apixaban $(n=1788)$ were initially evaluated (Figure 1). Before matching, the apixaban group were more likely to be older (mean age: $75.16 \pm 10.63$ years vs. 68.72 \pm 12.47 years) at the index date and had higher $\mathrm{CHA}_{2} \mathrm{DS}_{2}$ VASc $(3.92 \pm 1.70$ vs. $3.4 \pm 1.84)$ and HAS-BLED (3.02 \pm 1.36 vs. $2.55 \pm 1.43$ ) scores. Further, comorbid kidney disease was more prevalent in the apixaban group than in the warfarin group ( 23.71 vs. $13.72 \%$ ). However, the baseline mean eGFR was similar between the two groups.

After matching, the PS distributions were compatible and baseline characteristics were similar in the matched cohort, with each group involving 1,625 patients. The SMDs of all variables were $<0.1$ (Table 1). The mean age at the initiation of apixaban or warfarin was $74-75$ years. In total, 131 patients
$(8.06 \%)$ in the warfarin group and 119 patients $(7.32 \%)$ in the apixaban group had advanced CKD (i.e., eGFR $<30 \mathrm{ml} / \mathrm{min} / 1.73$ $\mathrm{m}^{2}$ ). Among the patients who received apixaban, 710 patients (56.31\%) and $913(43.69 \%)$ patients received a reduced and standard dose, respectively. The reduced and standard dose subgroups had a mean age of 78.5 years and 70 years, respectively (Supplementary Table 2). The patient characteristics before and after PS matching are shown in Table $\mathbf{1 .}$

\section{Study Outcomes}

The rates of stroke/SE, major bleeding, and in-hospital mortality are presented in Table 2. Compared to the warfarin group, the apixaban group showed significantly lower incidence rates of stroke/SE (10.77 vs. $7.08 \%, p<0.001)$, major bleeding (11.26 vs. $7.51 \%, p<0.001)$, and in-hospital any-cause death (5.84 vs. $3.94 \%, p=0.01$ ). The Kaplan-Meier curves (Figures 2A,C) also showed significant between-group differences in the cumulative incidence of stroke and major bleeding $(\log$-rank $p=0.01$ and $p=0.03$, respectively). Among the 250 patients with eGFR $<$ $30 \mathrm{ml} / \mathrm{min} / 1.73 \mathrm{~m}^{2}$ (Figures 2B,D), those treated with apixaban tended to have fewer events of stroke/SE and major bleeding (logrank $p=0.09$ and $p=0.06$, respectively). Meanwhile, there was no significant between-group difference in the rate of in-hospital any-cause death.

\section{Stroke/Systemic Embolism}

Cox proportional hazards regression analysis showed that apixaban treatment was associated with a lower risk of ischemic stroke/SE than warfarin treatment (adjusted hazard ratio $[\mathrm{aHR}]: 0.74 ; 95 \%$ confidence interval $[\mathrm{CI}]: 0.57-0.97 ; p$ $=0.03$ ) (Figure 3A, Supplementary Table 2). The relative effect on ischemic stroke/SE prevention was not influenced by the apixaban dose (standard dose: aHR, 0.71; 95\% CI, 0.50-1.01; p

TABLE 2 | Study outcomes in the matched cohort and in the advanced CKD subgroup.

\begin{tabular}{|c|c|c|c|c|c|c|c|c|}
\hline & \multicolumn{4}{|c|}{ Apixaban-warfarin matched cohort $(n=3,250)$} & \multicolumn{4}{|c|}{ Baseline eGFR $<30(n=250)$} \\
\hline & Event & Warfarin & Apixaban & $p$-value & Event & Warfarin & Apixaban & $p$-value \\
\hline Stroke/systemic embolism, $\boldsymbol{n}(\%)$ & 290 & $175(10.77)$ & $115(7.08)$ & 0.0002 & 21 & $15(11.45)$ & $6(5.04)$ & 0.0681 \\
\hline Ischemic or uncertain stroke & 222 & $111(6.83)$ & $111(6.83)$ & 1.0000 & 16 & $10(7.63)$ & $6(5.04)$ & 0.4031 \\
\hline Systemic embolism & 77 & $72(4.43)$ & $5(0.31)$ & $<.0001$ & 6 & $6(4.58)$ & $0(0.00)$ & \\
\hline Major bleeding, $\boldsymbol{n}(\%)$ & 305 & $183(11.26)$ & $122(7.51)$ & 0.0002 & 44 & $30(22.90)$ & $14(11.76)$ & 0.0209 \\
\hline Intracranial & 66 & $36(2.22)$ & $30(1.85)$ & 0.4556 & 6 & $4(3.05)$ & $2(1.68)$ & 0.4788 \\
\hline Ocular & 5 & $3(0.18)$ & $2(0.12)$ & 0.6545 & 0 & $0(0.00)$ & $0(0.00)$ & \\
\hline Intraabdominal & 2 & $2(0.12)$ & $0(0.00)$ & & 0 & $0(0.00)$ & $0(0.00)$ & \\
\hline Hematuria & 20 & $12(0.74)$ & $8(0.49)$ & 0.3696 & 2 & $1(0.76)$ & $1(0.84)$ & 0.9456 \\
\hline Gastrointestinal & 213 & $128(7.88)$ & $85(5.23)$ & 0.0023 & 36 & 25 (19.08) & $11(9.24)$ & 0.0269 \\
\hline Other sites & 8 & $7(0.43)$ & $1(0.06)$ & 0.0337 & 1 & $1(0.76)$ & $0(0.00)$ & \\
\hline \multicolumn{9}{|l|}{ Other outcomes, $n(\%)$} \\
\hline In-hospital death, $n$ (\%) & 159 & $95(5.85)$ & $64(3.94)$ & 0.0117 & 24 & $13(9.92)$ & $11(9.24)$ & 0.8554 \\
\hline Pneumonia & 302 & $175(10.77)$ & $127(7.82)$ & 0.0037 & 34 & $19(14.50)$ & $15(12.61)$ & 0.6618 \\
\hline Hip fracture & 26 & $17(1.05)$ & $9(0.55)$ & 0.1152 & 4 & $3(2.29)$ & $1(0.84)$ & 0.3616 \\
\hline
\end{tabular}

eGFR, estimated glomerular filtration rate, $\mathrm{ml} / \mathrm{min} / 1.73 \mathrm{~m}^{2}$. 


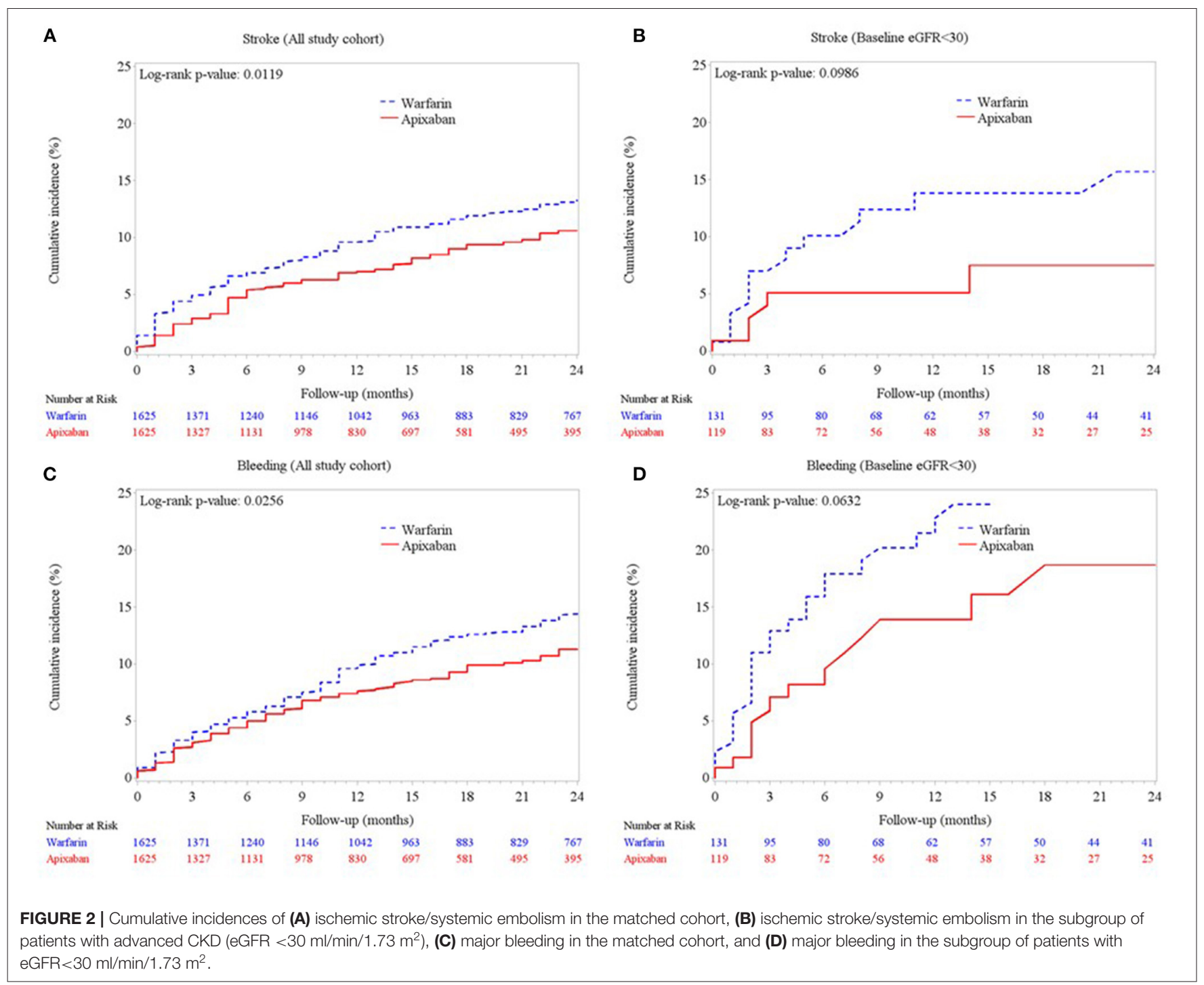

$=0.06$; reduced dose: $\mathrm{aHR}, 0.77 ; 95 \%$ CI, $0.57-1.05 ; p=0.09$ )

(Table 3, Supplementary Table 2)

\section{Major Bleeding}

In the entire cohort, gastrointestinal bleeding was high in both the apixaban and warfarin groups (5.23 vs. $7.88 \%)$, following by intracranial bleeding (1.85 vs. $2.22 \%)$ and hematuria (0.49 vs. $0.74 \%$ ) in Table 2. Apixaban reduced the risk of major bleeding by $22 \%$, but the difference did not reach statistical significance (aHR, 0.78; 95\% CI, 0.60-1.00; $p=0.05$ ). The standard dose of apixaban significantly lowered the risk of major bleeding (aHR, 0.66 ; $95 \%$ CI, $0.45-0.96 ; p=0.03$ ) than warfarin, but the reduced dose of apixaban didn't exhibit significantly difference in major bleeding (aHR, 0.84; 95\% CI, 0.63-1.12; $p=0.23$ ) (Table 3, Supplementary Table 3 ).

\section{Kidney Function}

The results according to the eGFR classification were consistent with the main analysis (Figures 3A,B). In the advanced CKD subgroup, apixaban initiation was significantly associated with a lower risk of stroke/SE (aHR: 0.63; 95\% CI: $0.40-0.98, p=$ 0.04 ), but not for major bleeding (aHR: 0.71 ; 95\% CI: 0.49$1.03 ; p=0.70$ ). Meanwhile, there was no significant difference in the risk of stroke/SE or major bleeding outcomes between apixaban and warfarin in the mild and moderate CKD subgroups. Further stratified analyses to investigate the impact of apixaban dose on the association between kidney function and risk of major bleeding showed inconclusive findings because there was no event in the advanced CKD subgroup (Table 3).

\section{Other Subgroup and Sensitivity Analyses}

The relative effects of apixaban according to a history of stroke and $\mathrm{CHA}_{2} \mathrm{DS}_{2}$-VASc score at baseline $(<4$ and $\geq 4)$, prior major bleeding, and HAS-BLED score at baseline $(<3$ and $\geq 3)$ are shown in Figures 3A,B. In general, apixaban was associated with more favorable outcomes than warfarin in patients without a history of stoke, CHA2DS2-VASc score $<4$, without history of 


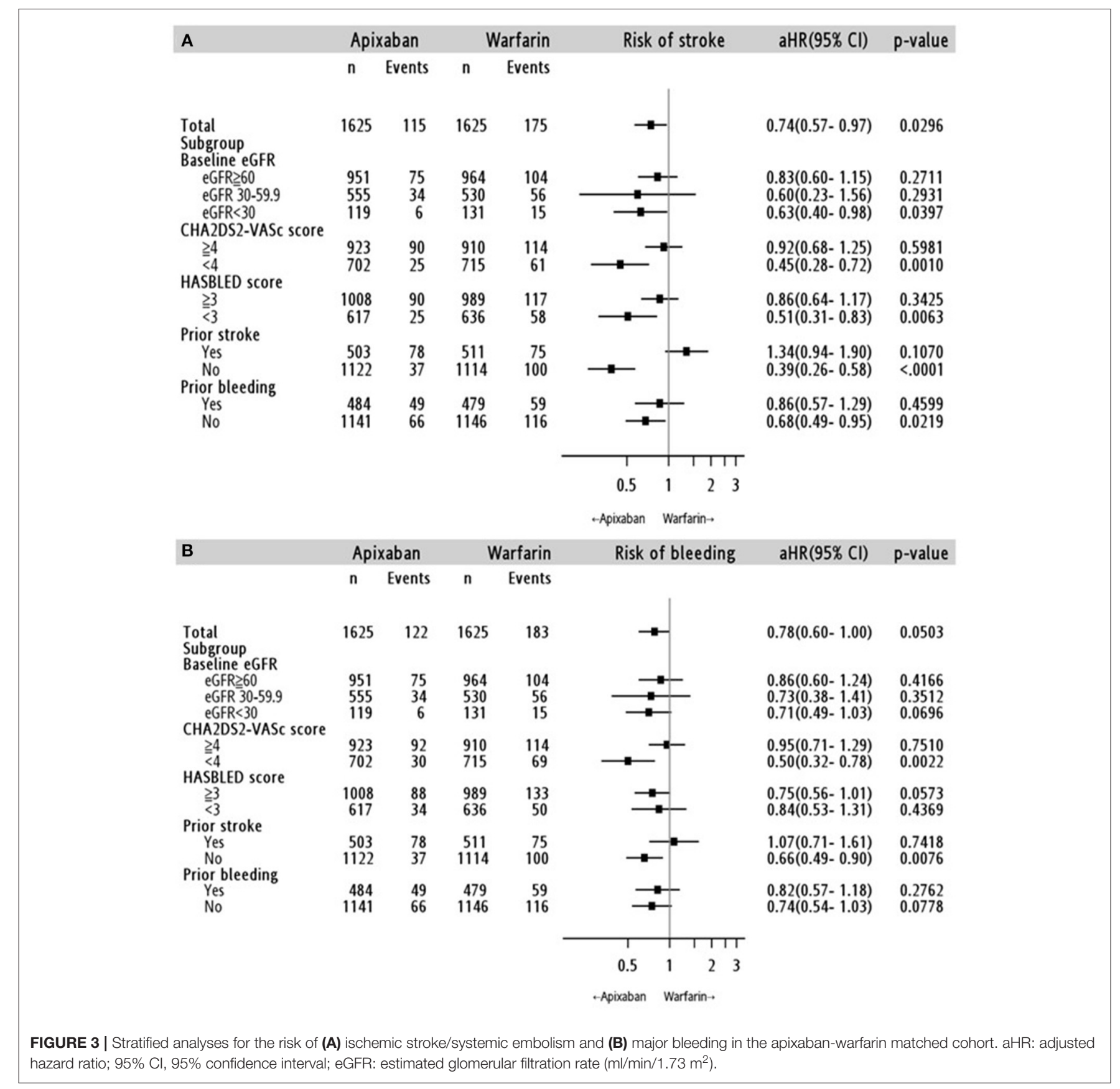

major bleeding, and with a HAS-BLED score $<3$. In addition, the choice of OAC did not increase the risk of pneumonia (aHR: 0.99; 95\% CI: 0.76-1.28; $p=0.91$ ) and hip fracture (aHR: 0.71; 95\% CI: 0.29-1.76; $p=0.44$ ) (Supplementary Table 4).

\section{DISCUSSION}

Real-world evidence on the benefit of apixaban in AF patients with CKD without dialysis is limited. This study found that apixaban lowers the risk of ischemic stroke or SE by $26 \%$ in AF patients with CKD and by $37 \%$ in those with advanced CKD.
Meanwhile, although the rate of major bleeding was lower in the apixaban group than it was in the warfarin group, the difference was not statistically significant in the overall cohort and across the eGFR groups. Subgroup analysis according to apixaban doses showed that a standard dose of $10 \mathrm{mg} /$ day was associated with a $34 \%$ lower risk of major bleeding.

The first real-word study on apixaban vs. warfarin use in CKD patients was published in 2017. The study, which included 146 patients with $\mathrm{CrCl}<25 \mathrm{~mL} / \mathrm{min}$ or serum creatinine $>2.5 \mathrm{mg} / \mathrm{dL}$, found no significant differences with respect to major bleeding or thromboembolic events between apixaban and 
TABLE 3 | Study outcomes by apixaban dose.

\begin{tabular}{|c|c|c|c|c|c|c|}
\hline & \multicolumn{3}{|c|}{$\begin{array}{c}\text { Apixaban (reduced dose)* } \\
\text { vs. Warfarin }\end{array}$} & \multicolumn{3}{|c|}{$\begin{array}{c}\text { Apixaban (standard dose)* } \\
\text { vs. Warfarin }\end{array}$} \\
\hline & aHR & $95 \% \mathrm{Cl}$ & $p$-value & aHR & $95 \% \mathrm{Cl}$ & $p$-value \\
\hline \multicolumn{7}{|l|}{ Stroke/SE } \\
\hline Overall & 0.77 & $(0.57,1.05)$ & 0.0955 & 0.71 & $(0.50,1.01)$ & 0.0575 \\
\hline \multicolumn{7}{|c|}{ Baseline eGFR group } \\
\hline$\geqq 60$ & 0.86 & $(0.57,1.27)$ & 0.3304 & 0.82 & $(0.54,1.20)$ & 0.3175 \\
\hline $30-59.9$ & 0.68 & $(0.42,1.09)$ & 0.2522 & 0.48 & $(0.20,1.00)$ & 0.0605 \\
\hline$<30$ & 0.66 & $(0.23,1.65)$ & 0.7231 & - & - & - \\
\hline \multicolumn{7}{|c|}{ Major bleeding } \\
\hline Overall & 0.84 & $(0.63,1.12)$ & 0.2286 & 0.66 & $(0.45,0.96)$ & 0.0287 \\
\hline \multicolumn{7}{|c|}{ Baseline eGFR group } \\
\hline$\geqq 60$ & 1.03 & $(0.67,1.57)$ & 0.5912 & 0.70 & $(0.43,1.11)$ & 0.0856 \\
\hline $30-59.9$ & 0.72 & $(0.47,1.07)$ & 0.1758 & 0.69 & $(0.36,1.21)$ & 0.2677 \\
\hline$<30$ & 0.81 & $(0.41,1.53)$ & 0.3807 & - & - & - \\
\hline
\end{tabular}

*Apixaban standard dose: $10 \mathrm{mg} /$ day, reduced dose: 2.5-5 mg/day; -: aHR was not available because no event was observed in the advanced CKD subgroup (eGFR $<30$ $\mathrm{ml} / \mathrm{min} / 1.73 \mathrm{~m}^{2}$ ). aHR: adjusted hazard ratio; 95\% Cl, 95\% confidence interval; eGFR: estimated glomerular filtration rate $\left(\mathrm{m} / \mathrm{min} / 1.73 \mathrm{~m}^{2}\right)$.

warfarin treatments (29). A recent subgroup analysis from the Apixaban for Reduction in Stroke and Other Thromboembolic Events in Atrial Fibrillation (ARISTOTLE) trial also showed no significant difference in stoke or SE prevention and all-cause mortality between apixaban and warfarin in patients with $\mathrm{CrCl}$ $25-30 \mathrm{~mL} / \mathrm{min}$ (30). A US Medicare population cohort of 22,739 AF patients with group 3, 4, and 5 CKD compared apixaban, rivaroxaban, and dabigatran with warfarin in CKD patients and found that apixaban was associated with the lowest risk of stroke/SE (31). However, most of the patients $(>80 \%)$ had group 3 CKD (eGFR between 30 and 59), and the patients were identified using diagnostic codes, limiting the generalizability of the findings to the advanced CKD patients. In the present study, the incidence rate of ischemic stroke/SE in patients with advanced CKD is comparable to that in previous observational studies $(18,29)$. The relatively large sample size of CKD patients in the present study and the data from a national representative database provide robust real-world evidence on the relative effect of apixaban in comparison to that of warfarin on stroke/SE prevention in a heterogeneous CKD population.

The apixaban dose is an important influencing factor of its efficacy and safety in patients with CKD. Although the apixaban label indicates a dose of $5 \mathrm{mg}$ twice daily for non-valvular AF, patients are recommended to take apixaban $2.5 \mathrm{mg}$ twice daily if they meet at least two of the following characteristics: age $\geq$ 80 years, body weight $\leq 60 \mathrm{~kg}$, and serum creatinine $\geq 1.5 \mathrm{mg} / \mathrm{dL}$ (32). In the secondary analyses of the ARISTOTLE trial, the risk of stroke/SE was $23 \%$ lower in standard dose apixaban $(5 \mathrm{mg}$ twice daily) than in warfarin, whereas there was no significant difference between reduced dose apixaban and warfarin (33). With respect to major bleeding, the risk was lower in apixaban than in warfarin irrespective of the apixaban dose, with the benefit being more profound in patients who were older, weighed less, and had serum creatinine $\geq 1.5 \mathrm{mg} / \mathrm{dL}$ (or lower $\mathrm{CrCl}$ ) (30). Apixaban was also reported to be associated with lower rates of major bleeding than warfarin among patients with $\mathrm{CrCl}$ of $25-30 \mathrm{ml} / \mathrm{min}$ (30). Overall, data from the ARISTOTLE trial support that standard dose apixaban (5 $\mathrm{mg}$ twice daily) has a better pharmacokinetic distribution in patients with $\mathrm{CrCl} 25-30$ $\mathrm{ml} / \mathrm{min}$ than in those with higher $\mathrm{CrCl}(>30 \mathrm{ml} / \mathrm{min})(30)$. The findings collectively suggest that the standard dose of apixaban may be safe in patients with CKD.

Given the low rate of OAC use in patients with eGFR $<15$ $\mathrm{ml} / \mathrm{min} / 1.73 \mathrm{~m}^{2}$ in the current study, we were unable to evaluate the relative benefits and disadvantages of apixaban in comparison to those of warfarin. However, the results support that the risk of stroke/SE was lower in apixaban treatment than in warfarin treatment in patients with eGFR $<30 \mathrm{ml} / \mathrm{min} / 1.73 \mathrm{~m}^{2}$, and apixaban was more beneficial in patients with low eGFR values than in those with high eGFR values, consistent with previous findings $(30,34)$. However, the effect of apixaban dose on the association between kidney function and risk of stroke/SE and major bleeding was not clarified in the present study.

The ARISTOLE trial suggested that apixaban was not inferior to warfarin as it had a mean time in therapeutic range (TTR) of $62 \%$ and an international normalized ratio (INR) of 2.0-3.0 (4). A subanalysis of the ARISTOTLE trial showed relatively lower mean TTR in East Asians (mean $27.2 \pm 11.07$ ) compared to those of non-East Asians (30.1 \pm 14.29$)$, and the duration with an international normalized ratio (INR) of $<2$ was longer in East Asians (28.6\%) than in non-East Asians (18\%) (35). Furthermore, the level of TTR varies between different countries (44-77\%), and according to a dabigatran multinational trial, the mean TTR was lowest (44\%) in Taiwan (36). In the present study, the mean INR was $1.97( \pm 1.01)$ during the total follow up period among patients with at least one INR values in the warfarin group ( $n$ $=1,511$ ), and these patients had a higher rate of intracranial hemorrhage compared to those of patients in the apixaban group. The high rate of intracranial hemorrhage in patients with a lower INR compared to those of controls is similar to the findings in Asian patients in the apixaban (35) and dabigatran multinational trials (36).

The effectiveness and safety of using warfarin is associated with its optimum therapeutic INR control. We noted a high rate of systematic embolism in patients treated with warfarin with great INR fluctuations from the mean value of $3.14( \pm 1.67)$ to $1.72( \pm 0.72)$ over the follow-up period (Supplementary Figure 1). The high variability of INR may be because of poor adherence or difficult management in some warfarin users $(35,37)$. Low intensity of anticoagulation is a common practice in Taiwan. Regarding the interpretation of these study results, it is important to address the differences in the relative effect of DOACs vs. warfarin between Asian and non-Asian populations $(35,36)$.

Of note, gastrointestinal bleeding and intracranial hemorrhage were the most common major bleedings in this study cohort, and this is consistent with the reports of a population-based observational study in Taiwan (1.81 per 100 person-years for gastrointestinal bleeding, 1.53 per 100 personyears for intracranial hemorrhage) (38). The population-based 
observational study and the meta-analyses of multinational randomized trials suggested that all DOACs can reduce overall major bleeding risk, but only apixaban was superior to warfarin in terms of fewer rates of major bleeding (39) or gastrointestinal bleeding (38). The reason for the differences in the risk of gastrointestinal bleeding between DOACs requires further research (40). In the present study, only patients with standarddose apixaban (vs. warfarin) revealed statistically significant reduction in overall major bleeding. Further research into precise apixaban dosing could support the use of apixaban as an alternative to warfarin in patients with chronic kidney disease and atrial fibrillation.

The present study has limitations. First, like other retrospective studies, biases due to residual confounding may not have been eliminated. The present study applied hospitalization for pneumonia and hip fracture as negative control outcomes to ensure the robustness of the relative effects of apixaban in comparison to those of warfarin. There were no associations between both negative control outcomes and treatment choices, which indicated that there was no evidence for unmeasured confounding bias. Second, there was a high proportion of patients who received reduced-dose apixaban. This could be because the patients were older, had worse kidney function, and a higher HAS-BLED score (Supplementary Table 5), as is characteristics of the Asian population $(41,42)$. Reduced-dose DOACs is common in real-world practice, especially in Asians (43-45). Third, the results may be applicable only in Taiwanese or Asian populations and have limited generalizability to the overall population of CKD patients. However, the clinical practice pattern in the study setting is likely to follow international clinical guidelines and could help improve understanding of the benefit/disadvantage of anticoagulation in patients with kidney dysfunction. Further, we measured kidney function using the Taiwan version of the MDRD formula (28), as is routine practice in Taiwan. MDRD-based eGFR values could be not the same as $\mathrm{CrCl}$ in ARISTOTLE trial (46). The current study findings may help establish the appropriate apixaban dose in high-risk patients, such as those with advanced CKD and the elderly, according to kidney function estimated with the MDRD formula.

In conclusion, the risk of stroke/SE is lower in AF patients receiving apixaban treatment than in those receiving warfarin treatment, and the benefits of apixaban are also noted in patients with advanced CKD (eGFR $<30 \mathrm{ml} / \mathrm{min} / 1.73 \mathrm{~m}^{2}$ ). Further, compared to warfarin, both standard and reduced dose of apixaban do not increase the risk of major bleeding. Our findings highlight the importance of appropriate anticoagulation treatment in patients with AF and kidney disease.

\section{REFERENCES}

1. Benjamin EJ, Muntner P, Alonso A, Bittencourt MS, Callaway CW, Carson AP, et al. Heart disease and stroke statistics-2019 update: a report from the american heart association. Circulation. (2019) 139:e56e528. doi: 10.1161/CIR.0000000000000659

\section{DATA AVAILABILITY STATEMENT}

The raw data supporting the conclusions of this article will be made available by the authors, without undue reservation.

\section{ETHICS STATEMENT}

The studies involving human participants were reviewed and approved by Institutional Review Board of Chang Gung Medical Foundation at Taipei, Taiwan. Written informed consent for participation was not required for this study in accordance with the national legislation and the institutional requirements.

\section{AUTHOR CONTRIBUTIONS}

C-MF, L-CL, and C-NH: conceptualization and wrote manuscript-original draft preparation. C-MF and C-NH: formal analysis, methodology, funding acquisition. All authors: investigation, validation, visualization, wrote manuscript-review, and editing.

\section{FUNDING}

This work was supported by the Ministry of Science and Technology, Taiwan, under Grants MOST 107-2622-E182A-001-CC3 and 109-2622-E-182A-001-CC3 (recipient: C-NH), and Kaohsiung Chang Gung Memorial Hospital, Taiwan under Grant CFRPG8J0041 (recipient: C-MF) and CMRPG8E0463 (recipient: C-NH).

\section{ACKNOWLEDGMENTS}

The authors thank Mrs. Chun-Hua Liao and Ying-Jen Hsu from the Department of Management of Information Systems, Chang Gung Memorial Foundation, for their technical assistance with electronic health records acquisition and data retrieval. We thank Ms. Hsiao-Ching Kuo from the Department of Pharmacy, Kaohsiung Chang Gung Memorial Hospital for the assistance on data management and statistical analysis.

\section{SUPPLEMENTARY MATERIAL}

The Supplementary Material for this article can be found online at: https://www.frontiersin.org/articles/10.3389/fcvm. 2021.752468/full\#supplementary-material

2. Connolly SJ, Ezekowitz MD, Yusuf S, Eikelboom J, Oldgren J, Parekh A, et al. Dabigatran versus warfarin in patients with atrial fibrillation. $N$ Engl J Med. (2009) 361:1139-51. doi: 10.1056/NEJMoa0905561

3. Giugliano RP, Ruff CT, Braunwald E, Murphy SA, Wiviott SD, Halperin JL, et al. Edoxaban versus warfarin in patients with atrial fibrillation. $N$ Engl J Med. (2013) 369:2093-104. doi: 10.1056/NEJMoa1310907 
4. Granger CB, Alexander JH, McMurray JJ, Lopes RD, Hylek EM, Hanna M, et al. Apixaban versus warfarin in patients with atrial fibrillation. $N$ Engl J Med. (2011) 365:981-92. doi: 10.1056/NEJMoa1107039

5. Patel MR, Mahaffey KW, Garg J, Pan G, Singer DE, Hacke W, et al. Rivaroxaban versus warfarin in nonvalvular atrial fibrillation. $N$ Engl J Med. (2011) 365:883-91. doi: 10.1056/NEJMoa1009638

6. Soliman EZ, Prineas RJ, Go AS, Xie D, Lash JP, Rahman M, et al. Chronic kidney disease and prevalent atrial fibrillation: the chronic renal insufficiency cohort (CRIC). Am Heart J. (2010) 159:1102-7. doi: 10.1016/j.ahj.2010.03.027

7. Alonso A, Lopez FL, Matsushita K, Loehr LR, Agarwal SK, Chen LY, et al. Chronic kidney disease is associated with the incidence of atrial fibrillation: the atherosclerosis risk in communities (ARIC) study. Circulation. (2011) 123:2946-53. doi: 10.1161/CIRCULATIONAHA.111.020982

8. Herzog CA, Asinger RW, Berger AK, Charytan DM, Díez J, Hart RG, et al. Cardiovascular disease in chronic kidney disease. a clinical update from kidney disease: improving global outcomes (KDIGO). Kidney Int. (2011) 80:572-86. doi: 10.1038/ki.2011.223

9. Wattanakit K, Cushman M, Stehman-Breen C, Heckbert SR, Folsom AR. Chronic kidney disease increases risk for venous thromboembolism. J Am Soc Nephrol. (2008) 19:135-40. doi: 10.1681/ASN.2007030308

10. Lutz J, Menke J, Sollinger D, Schinzel H, Thürmel K. Haemostasis in chronic kidney disease. Nephrol Dial Transplant. (2014) 29:2940. doi: $10.1093 / \mathrm{ndt} / \mathrm{gft} 209$

11. Bonde AN, Lip GY, Kamper AL, Hansen PR, Lamberts M, Hommel K, et al. Net clinical benefit of antithrombotic therapy in patients with atrial fibrillation and chronic kidney disease: a nationwide observational cohort study. J Am Coll Cardiol. (2014) 64:2471-82. doi: 10.1016/j.jacc.2014.09.051

12. Olesen JB, Lip GY, Kamper AL, Hommel K, Køber L, Lane DA, et al. Stroke and bleeding in atrial fibrillation with chronic kidney disease. N Engl J Med. (2012) 367:625-35. doi: 10.1056/NEJMoa1105594

13. Masson P, Kelly PJ, Craig JC, Lindley RI, Webster AC. Risk of stroke in patients with ESRD. Clin J Am Soc Nephrol. (2015) 10:158592. doi: $10.2215 / C J N .12001214$

14. Limdi NA, Beasley TM, Sun J, Stockbridge N, Pacanowski M, Florian J. Thromboembolic and hemorrhagic outcomes in the direct oral anticoagulant trials across the spectrum of kidney function. Clin Pharmacol Ther. (2020) 109:1593-605. doi: 10.1002/cpt.2131

15. Aarnio E, Huupponen R, Korhonen MJ. Important factors affecting the choice of an oral anticoagulant may be missed in database studies. J Intern Med. (2018) 283:214-5. doi: 10.1111/joim.12686

16. Steinberg BA, Shrader P, Thomas L, Ansell J, Fonarow GC, Gersh BJ, et al. Factors associated with non-vitamin $\mathrm{K}$ antagonist oral anticoagulants for stroke prevention in patients with new-onset atrial fibrillation: results from the outcomes registry for better informed treatment of atrial fibrillation II (ORBIT-AF II). Am Heart J. (2017) 189:40-7. doi: 10.1016/j.ahj.2017.03.024

17. Wang X, Tirucherai G, Marbury TC, Wang J, Chang M, Zhang D, et al. Pharmacokinetics, pharmacodynamics, and safety of apixaban in subjects with end-stage renal disease on hemodialysis. J Clin Pharmacol. (2016) 56:62836. doi: $10.1002 /$ jcph. 628

18. Siontis KC, Zhang X, Eckard A, Bhave N, Schaubel DE, He K, et al. Outcomes associated with apixaban use in patients with end-stage kidney disease and atrial fibrillation in the United States. Circulation. (2018) 138:151929. doi: 10.1161/CIRCULATIONAHA.118.035418

19. Mavrakanas TA, Garlo K, Charytan DM. Apixaban versus no anticoagulation in patients undergoing long-term dialysis with incident atrial fibrillation. Clin J Am Soc Nephrol. (2020) 15:1146-54. doi: 10.2215/CJN.11650919

20. Shao SC, Chan YY, Yang YHK, Lin SJ, Hung MJ, Chien RN, et al. The chang gung research database-a multi-institutional electronic medical records database for real-world epidemiological studies in Taiwan. Pharmacoepidemiol Drug Saf. (2019) 28:593-600. doi: 10.1002/pds.4713

21. Tsai MS, Lin MH, Lee CP, Yang YH, Chen WC, Chang GH, et al. Chang gung research database: a multi-institutional database consisting of original medical records. Biomed J. (2017) 40:263-9. doi: 10.1016/j.bj.2017.08.002

22. Chang S-H, Chou I-J, Yeh Y-H, Chiou M-J, Wen M-S, Kuo C-T, et al. Association between use of non-vitamin $\mathrm{K}$ oral anticoagulants with and without concurrent medications and risk of major bleeding in nonvalvular atrial fibrillation. Jama. (2017) 318:1250-9. doi: 10.1001/jama.201 7.13883
23. Austin PC. The use of propensity score methods with survival or timeto-event outcomes: reporting measures of effect similar to those used in randomized experiments. Stat Med. (2014) 33:1242-58. doi: 10.1002/sim.5984

24. Rubin DB. Using propensity scores to help design observational studies: application to the tobacco litigation. Health Serv Outcomes Res Methodol. (2001) 2:169-88. doi: 10.1023/A:1020363010465

25. Sundararajan V, Henderson T, Perry C, Muggivan A, Quan H, Ghali WA. New ICD-10 version of the charlson comorbidity index predicted in-hospital mortality. J Clin Epidemiol. (2004) 57:1288-94. doi: 10.1016/j.jclinepi.2004.03.012

26. Parsons LS. Performing A 1:N Case-Control Match On Propensity Score. Seattle, Washington: SAS Users Group International (2001). p. 165-29.

27. Austin PC. Balance diagnostics for comparing the distribution of baseline covariates between treatment groups in propensity-score matched samples. Stat Med. (2009) 28:3083-107. doi: 10.1002/sim.3697

28. Chen L-I, Guh J-Y, Wu K-D, Chen Y-M, Kuo M-C, Hwang S-J, et al. Modification of diet in renal disease (MDRD) study and CKD epidemiology collaboration (CKD-EPI) equations for Taiwanese adults. PLoS ONE. (2014) 9:e99645. doi: 10.1371/journal.pone.0099645

29. Stanton BE, Barasch NS, Tellor KB. Comparison of the Safety and effectiveness of apixaban versus warfarin in patients with severe renal impairment. Pharmacotherapy. (2017) 37:412-9. doi: 10.1002/phar.1905

30. Stanifer JW, Pokorney SD, Chertow GM, Hohnloser SH, Wojdyla DM, Garonzik S, et al. Apixaban versus warfarin in patients with atrial fibrillation and advanced chronic kidney disease. Circulation. (2020) 141:138492. doi: 10.1161/CIRCULATIONAHA.119.044059

31. Wetmore JB, Roetker NS, Yan H, Reyes JL, Herzog CA. Directacting oral anticoagulants versus warfarin in medicare patients with chronic kidney disease and atrial fibrillation. Stroke. (2020) 51:236473. doi: 10.1161/STROKEAHA. 120.028934

32. Food and Drug Administration. Eliquis 2012. (2019). Availableonline at: https://www.accessdata.fda.gov/drugsatfda_docs/label/2019/ 202155s021lbl.pdf.

33. Alexander JH, Andersson U, Lopes RD, Hijazi Z, Hohnloser SH, Ezekowitz JA, et al. Apixaban $5 \mathrm{mg}$ twice daily and clinical outcomes in patients with atrial fibrillation and advanced age, low body weight, or high creatinine: a secondary analysis of a randomized clinical trial. JAMA Cardiol. (2016) 1:673-81. doi: 10.1001/jamacardio.2016.1829

34. Ashley J, McArthur E, Bota S, Harel Z, Battistella M, Molnar AO, et al. Risk of cardiovascular events and mortality among elderly patients with reduced gfr receiving direct oral anticoagulants. Am J Kidney Dis. (2020) 76:311-20. doi: 10.1053/j.ajkd.2020.02.446

35. Goto S, Zhu J, Liu L, Oh BH, Wojdyla DM, Aylward P, et al. Efficacy and safety of apixaban compared with warfarin for stroke prevention in patients with atrial fibrillation from East Asia: a subanalysis of the apixaban for reduction in stroke and other thromboembolic events in atrial fibrillation (ARISTOTLE) trial. Am Heart J. (2014) 168:303-9. doi: 10.1016/j.ahj.2014.06.005

36. Wallentin L, Yusuf S, Ezekowitz MD, Alings M, Flather M, Franzosi MG, et al. Efficacy and safety of dabigatran compared with warfarin at different levels of international normalised ratio control for stroke prevention in atrial fibrillation: an analysis of the RE-LY trial. Lancet. (2010) 376:97583. doi: 10.1016/S0140-6736(10)61194-4

37. Shen AY, Yao JF, Brar SS, Jorgensen MB, Chen W. Racial/ethnic differences in the risk of intracranial hemorrhage among patients with atrial fibrillation. $J$ Am Coll Cardiol. (2007) 50:309-15. doi: 10.1016/j.jacc.2007.01.098

38. Chan YH, Lee HF, See LC, Tu HT, Chao TF, Yeh YH, et al. Effectiveness and safety of four direct oral anticoagulants in asian patients with nonvalvular atrial fibrillation. Chest. (2019) 156:529-43. doi: 10.1016/j.chest.2019.04.108

39. Ruff CT, Giugliano RP, Braunwald E, Hoffman EB, Deenadayalu N, Ezekowitz $\mathrm{MD}$, et al. Comparison of the efficacy and safety of new oral anticoagulants with warfarin in patients with atrial fibrillation: a meta-analysis of randomised trials. Lancet. (2014) 383:955-62. doi: 10.1016/S0140-6736(13)62343-0

40. Feagins LA, Weideman RA. GI bleeding risk of doacs versus warfarin: is newer better? Dig Dis Sci. (2018) 63:1675-7. doi: 10.1007/s10620-018-5060-1

41. Lip GY, Wang KL, Chiang CE. Non-vitamin K antagonist oral anticoagulants (NOACs) for stroke prevention in Asian patients with atrial fibrillation: time for a reappraisal. Int J Cardiol. (2015) 180:246-54. doi: 10.1016/j.ijcard.2014.11.182 
42. Chao TF, Chen SA, Ruff CT, Hamershock RA, Mercuri MF, Antman EM, et al. Clinical outcomes, edoxaban concentration, and anti-factor $\mathrm{Xa}$ activity of Asian patients with atrial fibrillation compared with nonAsians in the ENGAGE AF-TIMI 48 trial. Eur Heart J. (2019) 40:151827. doi: 10.1093/eurheartj/ehy807

43. Laugesen EK, Staerk L, Carlson N, Kamper AL, Olesen JB, Torp-Pedersen $\mathrm{C}$, et al. Non-vitamin $\mathrm{K}$ antagonist oral anticoagulants vs. vitamin-K antagonists in patients with atrial fibrillation and chronic kidney disease: a nationwide cohort study. Thromb J. (2019) 17:21. doi: 10.1186/s12959-019-0 211-y

44. Chan YH, Chao TF, Chen SW, Lee HF, Yeh YH, Huang YC, et al. Offlabel dosing of non-vitamin $\mathrm{K}$ antagonist oral anticoagulants and clinical outcomes in Asian patients with atrial fibrillation. Heart Rhythm. (2020) 17:2102-10. doi: 10.1016/j.hrthm.2020.07.022

45. Ono T, Kohsaka S, Takatsuki S, Fukuda K, Suzuki M. Inconsistent dosing of non-vitamin $\mathrm{K}$ oral anticoagulants: differences between the United States and Japan. J Am Coll Cardiol. (2017) 70:118. doi: 10.1016/j.jacc.201 7.03.609

46. Hohnloser SH, Hijazi Z, Thomas L, Alexander JH, Amerena J, Hanna M, et al. Efficacy of apixaban when compared with warfarin in relation to renal function in patients with atrial fibrillation: insights from the ARISTOTLE trial. Eur Heart J. (2012) 33:2821-30. doi: 10.1093/eurheartj/ehs274

Conflict of Interest: The authors declare that the research was conducted in the absence of any commercial or financial relationships that could be construed as a potential conflict of interest.

Publisher's Note: All claims expressed in this article are solely those of the authors and do not necessarily represent those of their affiliated organizations, or those of the publisher, the editors and the reviewers. Any product that may be evaluated in this article, or claim that may be made by its manufacturer, is not guaranteed or endorsed by the publisher.

Copyright (c) $2021 \mathrm{Fu}$, Li, Lee, Wang and Hsu. This is an open-access article distributed under the terms of the Creative Commons Attribution License (CC BY). The use, distribution or reproduction in other forums is permitted, provided the original author(s) and the copyright owner(s) are credited and that the original publication in this journal is cited, in accordance with accepted academic practice. No use, distribution or reproduction is permitted which does not comply with these terms. 